\title{
Antifeedant Activities of Lignans from Stem Bark of Zanthoxylum armatum DC. against Tribolium castaneum
}

\author{
Wenjuan Zhang ${ }^{1}$, Yang Wang ${ }^{1}$, Zhufeng Geng ${ }^{1,2}$, Shanshan Guo ${ }^{1}$, Juqin Cao ${ }^{1}$, Zhe Zhang ${ }^{1}$, \\ Xue Pang ${ }^{1}$, Zhenyang Chen ${ }^{1}$, Shushan Du ${ }^{1, *}$ and Zhiwei Deng ${ }^{2}$ \\ 1 Beijing Key Laboratory of Traditional Chinese Medicine Protection and Utilization, \\ Faculty of Geographical Science, Beijing Normal University, No. 19 Xinjiekouwai Street, Beijing 100875, \\ China; zwj0729@mail.bnu.edu.cn (W.Z.); wangyangjs@mail.bnu.edu.cn (Y.W.); \\ gengzhufeng@bnu.edu.cn (Z.G.); guoshanshan@mail.bnu.edu.cn (S.G.); juqincao@mail.bnu.edu.cn (J.C.); \\ zhezhang@mail.bnu.edu.cn (Z.Z.); pangxue0924@mail.bnu.edu.cn (X.P.); sunnychen@mail.bnu.edu.cn (Z.C.) \\ 2 Analytical and Testing Center, Beijing Normal University, No. 19 Xinjiekouwai Street, Haidian District, \\ Beijing 100875, China; dengzw@bnu.edu.cn \\ * Correspondence: dushushan@bnu.edu.cn; Tel.: +86-10-6220-8022
}

Received: 24 January 2018; Accepted: 7 March 2018; Published: 9 March 2018

\begin{abstract}
The speciation of a methanolic extract of Zanthoxylum armatum stem bark has enabled the isolation and characterization of 11 known lignans. Among them, five compounds (6, 8-11) are reported in this plant for the first time. All of the chemical structures were elucidated on the basis of NMR spectral analysis. Additionally, their antifeedant activities against Tribolium castaneum were evaluated scientifically. Among them, asarinin (1), with an $\mathrm{EC}_{50}$ of $25.64 \mathrm{ppm}$, exhibited a much stronger antifeedant activity than the positive control, toosendanin $\left(\mathrm{EC}_{50}=71.69 \mathrm{ppm}\right)$. Moreover, fargesin (2), horsfieldin (3), and magnolone (10), with $\mathrm{EC}_{50}$ values of 63.24, 68.39, and $78.37 \mathrm{ppm}$, showed almost the same antifeedant activity as the positive control. From the perspective of structure-effectiveness relationship, compounds with the chemical group of methylenedioxy exhibited higher antifeedant activities and have potential to be developed into novel antifeedants or potential lead compounds to protect food and crops in storage.
\end{abstract}

Keywords: Zanthoxylum armatum; Tribolium castaneum; antifeedant activity; chemical constituents; methylenedioxy

\section{Introduction}

Tribolium castaneum, the red flour beetle, is one of the most destructive storage pests and has a direct effect on the reduction of the quantity and quality of grain during postharvest storage [1]. According to reports, the damages to stored grains and their related grain products by storage pests could be as high as $20-30 \%$ in tropical countries [2]. As an invertebrate model organism, T. castaneum is used in various biological disciplines, including developmental biology, immunology, evolutionary studies, and ecology [3-5]. Nowadays, synthetic insecticides have been commonly and heavily used to protect stored grains and their related products against pests. However, massive and repeated usage of synthetic insecticides has resulted in a series of problems, including pesticide residue, environmental pollution, and insect resistance [6,7]. Therefore, increased attention has been given to develop alternative strategies which include using plant-derived compounds as effective natural anti-insect agents or leading compounds for the control of insects [6,8]. Plants containing bioactive compounds could provide an alternative source of insect control agents and many of them are selective and have little or no harmful effects on the environment and non-targeted organisms $[9,10]$. 
Zanthoxylum armatum DC., belonging to the family Rutaceae, is a traditional evergreen shrub that is distributed in Southeast Asia [11]. In China, it is widely cultivated in the South of China, especially in Sichuan province [12]. As a Chinese traditional folk medicinal herb, Z. armatum is applied for toothaches, stomachaches, and wet sores [13]. According to reports, the chemical composition category of Z. armatum is various, and including alkaloids, saponins, phenolic glycosides, coumarins, lignans, sterols, flavonoids, and terpenes [14-19]. Different chemical constituents result in different bioactivities, such as larvicidal [18], insecticidal [20], antioxidant [21,22], antibacterial [23], cytotoxic [24,25], antimicrobial [11], and anthelmintic [26] activities. In our present screening program, 11 lignans were isolated from Zanthoxylum armatum and some of them showed significant antifeedant activity. Moreover, their antifeedant activities were evaluated for the first time.

\section{Results}

In total, 11 known lignans, asarinin (1), fargesin (2), horsfieldin (3), eudesmin (4), planispine A (5), planispine B (6), yangambin (7), acuminatolide (8), salicifoliol (9), magnolone (10), and magnone A (11), were isolated from the methanol extract of Z. armatum through a variety of column chromatography and preparative HPLC processes. Their chemical structures are displayed in Figure 1. Among them, five compounds, planispine B (6), acuminatolide (8), salicifoliol (9), magnolone (10), and magnone A (11), were isolated from this plant for first time. The structural characteristics of the 11 lignans are obvious. As shown in Figure 1, compounds 1-7, compounds 8-9, and compounds 10-11 each have the same basic mother nucleus. Compared with compounds 1-7, the basic mother nucleus of compounds 8-9 only has one benzene cycle on one side, while for compounds 8-9, one of the furan cycles on the mother nucleus is opened. Therefore, different chemical structures with different substituents may result in different bioactivities.
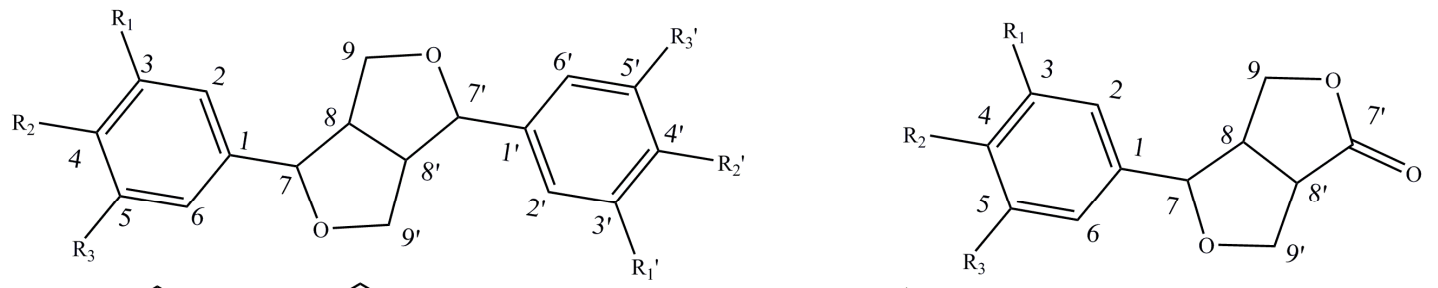

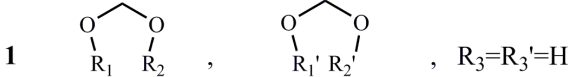

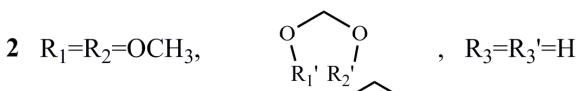

$$
\begin{aligned}
& 3 \mathrm{R}_{1}=\mathrm{OH}, \quad \mathrm{R}_{2}=\mathrm{OCH}_{3}, \quad l_{\mathrm{R}_{1}^{\prime} \mathrm{R}_{2}^{\prime}}^{\mathrm{O}}, \mathrm{R}_{3}=\mathrm{R}_{3}^{\prime}=\mathrm{H} \\
& 4 \mathrm{R}_{1}=\mathrm{R}_{2}=\mathrm{R}_{1}^{\prime}=\mathrm{R}_{2}{ }^{\prime}=\mathrm{OCH}_{3}, \quad \mathrm{R}_{3}=\mathrm{R}_{3}^{\prime}=\mathrm{H} \\
& 5 \quad \mathrm{R}_{1}=\mathrm{R}_{1}^{\prime}=\mathrm{OCH}_{3}, \mathrm{R}_{2}=\mathrm{O}{ }_{1 " \prime}^{\prime \prime \prime}{ }^{\prime \prime}, \mathrm{R}_{3}=\mathrm{R}_{3}{ }^{\prime \prime}=\mathrm{H}, \mathrm{R}_{2}^{\prime}=\mathrm{OH}
\end{aligned}
$$

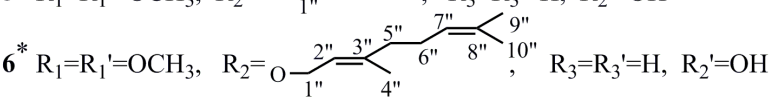

$$
\begin{aligned}
& 7 \mathrm{R}_{1}=\mathrm{R}_{2}=\mathrm{R}_{3}=\mathrm{R}_{1}^{\prime}=\mathrm{R}_{2}^{\prime}=\mathrm{R}_{3}^{\prime}=\mathrm{OCH}_{3}
\end{aligned}
$$
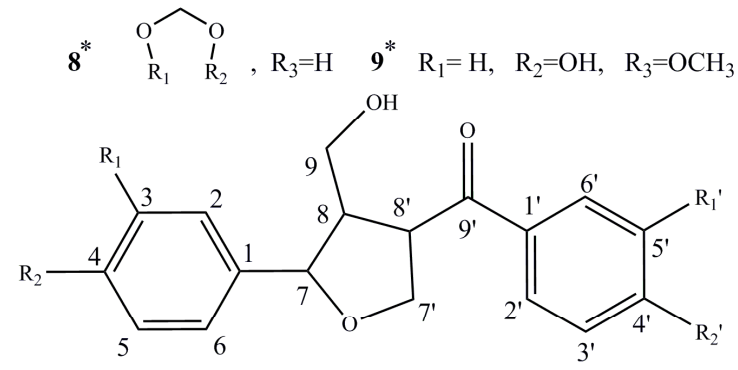

$$
\begin{aligned}
& 10 * \stackrel{\mathrm{R}}{\mathrm{R}}_{\mathrm{R}_{1}}^{\stackrel{\mathrm{O}}{\mathrm{R}_{2}}}, \mathrm{R}_{1}^{\prime}=\mathrm{R}_{2}{ }^{\prime}=\mathrm{OCH}_{3} \\
& 11^{*} \mathrm{R}_{1}=\mathrm{R}_{2}=\mathrm{R}_{1}^{\prime}=\mathrm{R}_{2}^{\prime}=\mathrm{OCH}_{3}
\end{aligned}
$$

Figure 1. Chemical structures of compounds 1-11. * represents known but derived from the plant of Zanthoxylum armatum for the first time.

The antifeedant activities of the 11 lignans displayed in Figure 1 were measured, and the results are presented in Table 1. At the lowest concentration of 25 ppm, asarinin (1) exhibited the highest antifeedant activity against T. castaneum with an antifeedant index of $49.45 \%$. Furthermore, fargesin (2), acuminatolide (8), magnolone (10), yangambin (7), eudesmin (4), and horsfieldin (3) also showed higher antifeedant activities against T. castaneum than the positive control, toosendanin, at the lowest concentration of 25 ppm, with antifeedant indices of $40.65 \%, 39.15 \%, 38.93 \%, 37.80 \%, 35.73 \%$, 
and $34.99 \%$, respectively. The $\mathrm{EC}_{50}$ of 10 lignans are displayed in Figure 2. As shown in Figure 2, eudesmin (4), planispine B (6), yangambin (7), acuminatolide (8), salicifoliol (9), and magnone A (11) exhibited significant differences compared to the positive control, toosendanin, at $p<0.05$. Meanwhile, fargesin (2), horsfieldin (3), and magnolone (10) showed nearly the same antifeedant level against $T$. castaneum. Asarinin (1) was the only compound that exhibited a lower $\mathrm{EC}_{50}$ than the positive control with the significant difference level at $p<0.05$.

Table 1. Antifeedant activities of lignans from stem bark of Zanthoxylum armatum.

\begin{tabular}{ccccccc}
\hline \multirow{2}{*}{ Compound } & \multicolumn{5}{c}{ Antifeedant Index (\%, Mean $\pm \mathbf{S D )}$} & \multirow{2}{*}{ EC $_{\mathbf{5 0}}$ (ppm) } \\
\cline { 2 - 6 } & $\mathbf{2 5} \mathbf{~ p p m}$ & $\mathbf{7 4} \mathbf{~ p p m}$ & $\mathbf{2 2 2} \mathbf{~ p ~ p m}$ & $\mathbf{6 6 7} \mathbf{~ p ~ p m ~}$ & $\mathbf{2 0 0 0} \mathbf{~ p p m}$ & \\
\hline Asarinin (1) & $49.45 \pm 3.78$ & $55.51 \pm 1.79$ & $68.03 \pm 4.22$ & $70.72 \pm 2.83$ & $75.83 \pm 3.69$ & $25.64 \pm 3.21$ \\
Fargesin (2) & $40.65 \pm 4.51$ & $52.12 \pm 2.31$ & $60.71 \pm 3.21$ & $75.21 \pm 1.39$ & $81.96 \pm 2.93$ & $63.24 \pm 4.83$ \\
Horsfieldin (3) & $34.99 \pm 5.32$ & $48.91 \pm 3.37$ & $73.74 \pm 3.04$ & $73.79 \pm 6.01$ & $81.12 \pm 3.33$ & $68.39 \pm 5.58$ \\
Eudesmin (4) & $35.73 \pm 4.37$ & $41.28 \pm 3.07$ & $43.57 \pm 2.16$ & $55.97 \pm 3.46$ & $69.82 \pm 3.56$ & $245.72 \pm 3.74$ \\
Planispine (5) & - & - & - & - & - & - \\
Planispine B (6) & $29.76 \pm 3.16$ & $45.95 \pm 5.11$ & $47.79 \pm 3.27$ & $58.38 \pm 3.17$ & $68.64 \pm 5.23$ & $219.84 \pm 4.69$ \\
Yangambin (7) & $37.80 \pm 3.12$ & $49.71 \pm 2.61$ & $50.82 \pm 4.27$ & $60.95 \pm 3.26$ & $66.96 \pm 4.16$ & $132.50 \pm 3.61$ \\
Acuminatolide (8) & $39.15 \pm 1.21$ & $41.36 \pm 3.83$ & $54.51 \pm 2.18$ & $77.35 \pm 3.65$ & $74.76 \pm 3.93$ & $102.12 \pm 2.40$ \\
Salicifoliol (9) & $6.82 \pm 3.47$ & $12.52 \pm 2.15$ & $24.78 \pm 3.17$ & $30.46 \pm 5.23$ & $39.06 \pm 4.17$ & $1069.20 \pm 3.83$ \\
Magnolone (10) & $38.93 \pm 4.29$ & $47.52 \pm 1.91$ & $62.16 \pm 3.93$ & $71.94 \pm 3.19$ & $74.14 \pm 3.95$ & $78.37 \pm 3.44$ \\
Magnone A (11) & $21.66 \pm 3.91$ & $28.99 \pm 3.85$ & $32.76 \pm 4.33$ & $43.53 \pm 2.77$ & $56.60 \pm 5.06$ & $1203.53 \pm 3.95$ \\
Toosendanin * & $32.32 \pm 2.28$ & $52.45 \pm 3.27$ & $69.52 \pm 2.47$ & $76.54 \pm 3.62$ & $86.27 \pm 3.51$ & $71.69 \pm 3.13$ \\
\hline
\end{tabular}

* represents the positive control. The antifeedant index of the blank control is 0 .

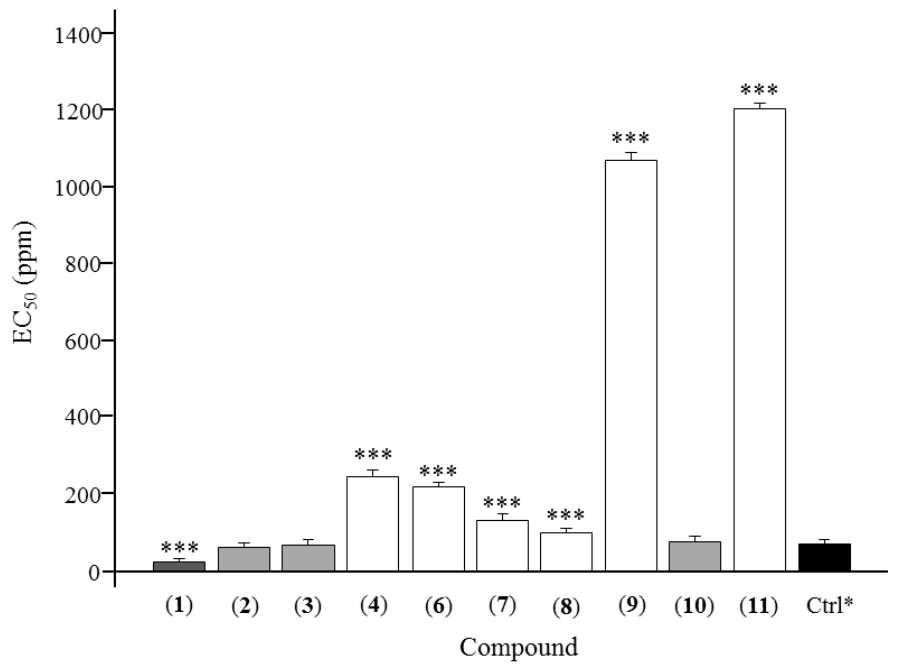

Figure 2. Antifeedant $\mathrm{EC}_{50}$ of 10 lignans from Zanthoxylum armatum. * represents the positive control, toosendanin. ${ }^{* * *}$ represents the significant difference at $p<0.05$.

\section{Discussion}

Compared with traditional isolation method, the application of preparative HPLC provided an effective way to achieve the isolation and purification of compounds. Moreover, this method provides a basis for the establishment of the method of compound separation and purification. After that, 11 compounds were identified as lignan compounds. As reported in the literature [27], lignans are a large group of natural products, and many of them exhibit potential bioactivities with antioxidant, antitumor, anti-inflammatory, and antiviral properties. Here, the antifeedant activities of lignans isolated from $Z$. armatum were investigated.

As shown in Table 1, there was a dose-dependent antifeedant activity, and the antifeedant activity increased with the increase of the concentration gradient from 25 ppm to 2000 ppm. Most 
of the lignans exhibited antifeedant activities against T. castaneum. At a concentration of $74 \mathrm{ppm}$, asarinin (1) and fargesin (2) showed high antifeedant activities with an antifeedant index above $50 \%$. At a concentration of $222 \mathrm{ppm}$, asarinin (1), fargesin (2), horsfieldin (3), and magnolone (10) exhibited higher antifeedant activities than other lignans. Moreover, asarinin (1) and fargesin (2) showed nearly the same antifeedant index as the positive control, toosendanin, at a concentration of $2000 \mathrm{ppm}$. According to the antifeedant results at different concentrations, asarinin (1) is the most active compound, which has potential to be developed into novel antifeedant formulations for insect control in storage. In Figure 2, asarinin (1), with an $\mathrm{EC}_{50}$ of $25.64 \mathrm{ppm}$, exhibited a significant difference compared to the positive control, toosendanin $\left(\mathrm{EC}_{50}=71.69 \mathrm{ppm}\right)$ at $p<0.05$. What is more, fargesin (2), horsfieldin (3), and magnolone (10) showed the same antifeedant activity as the positive control, with $\mathrm{EC}_{50}$ values of $63.24,68.39$, and $78.37 \mathrm{ppm}$, respectively. Meanwhile, eudesmin (4), planispine B (6), yangambin (7), and acuminatolide (8), with $\mathrm{EC}_{50}$ values of 245.72, $219.84,132.50$, and $102.12 \mathrm{ppm}$, respectively, showed less antifeedant activity compared to the positive control at $p<0.05$. Salicifoliol (9) $\left(\mathrm{EC}_{50}=1069.20 \mathrm{ppm}\right)$ and magnone $\mathrm{A}(\mathbf{1 1})\left(\mathrm{EC}_{50}=1203.53 \mathrm{ppm}\right)$ exhibited much less antifeedant activity, and planispine A (5) showed no antifeedant activity. From the perspective of structure-effectiveness relationship, asarinin (1), having two methylenedioxy groups, showed the highest antifeedant activity. Moreover, fargesin (2), horsfieldin (3), magnolone (10), and acuminatolide (8), each having one group of methylenedioxy, displayed less antifeedant activity than asarinin (1). Salicifoliol (9), magnone A (11), and planispine A (5), having no methylenedioxy groups, showed much less or no antifeedant activity. As it was reported, compounds with methylenedioxy groups have various bioactivities, such as improving skin condition, antioxidant activity [28], anti-inflammatory activity [29], radical scavenging activity [30], insecticidal activity [31], antineuroinflammatory effects [32], antiparasitic activity, and anti-osteoporotic activity [33]. Therefore, methylenedioxy, as an active chemical group, may play a key role in antifeedant activities. Moreover, compounds with one or more methylenedioxy groups might be developed into potential lead compounds or conventional antifeedant alternatives. Since the natural resources of Z. armatum are abundant, it is meaningful to take advantage of its non-medicinal parts to achieve a comprehensive utilization of resources.

\section{Materials and Methods}

\subsection{General Information}

NMR spectra were recorded on a Bruker Avance III 500 instrument (Bruker-Biospin, Billerica, MA, USA) at room temperature. Preparative HPLC was performed on a Waters Delta Prep 4000 system, which was equipped with a Waters 2487 dual $\lambda$ absorbance detector. A Rainbow Kromasil- $C_{18}$ $(10 \mathrm{~mm} \times 250 \mathrm{~mm}, 10 \mu \mathrm{m})$ column was selected and performed for preparative HPLC. Column chromatography was performed on silica gel (160-200 mesh) and Thin Layer Chromatography (TLC) analysis was carried out on silica gel G plates (Qingdao Marine Chemical Plant, Qingdao, China). Sephadex LH-20 was purchase from Amersham Pharmacia Biotech (Beijing, China). Mitsubishi Chemical Ion (MCI) gel CHP20P (75-150 $\mu \mathrm{m}$ ) was supplied by Kaiteki Company (Tokyo, Japan). All of the analytical reagents were purchased from Beijing Chemical Factory (Beijing, China). The deuterated solvents $\left(\mathrm{CDCl}_{3}\right.$, Deuterated ratio, $\left.99.8 \%\right)$ with tetramethylsilane (TMS) were purchased from Cambridge Isotope Laboratories, Inc. (Andover, MA, USA).

\subsection{Material}

\subsubsection{Plants}

The fresh stem bark of Z. armatum was collected in Wen county, Gansu Province, China (32.95 N latitude, $104.70^{\circ}$ E longitude) on 2 September 2015. The plant was identified by Dr. Liu, Q.R. (College of Life Sciences, Beijing Normal University, Beijing, China) and the voucher 
specimen (BNU-CMH-DuSS-2015-09-02-001) was deposited at the Herbarium (BNU) of the Faculty of Geographical Science, Beijing Normal University.

\subsubsection{Insects}

The red flour beetle, Tribolium castaneum, was identified by Liu, Z.L. (College of Plant Protection, China Agricultural University, Beijing, China). A laboratory culture of T. castaneum followed the same method mentioned in the recent literature [34]. The red flour beetle was maintained in the dark cabinet of an incubator at $29-30{ }^{\circ} \mathrm{C}$ and $70-80 \%$ relative humidity (RH). Glass containers $(0.5 \mathrm{~L})$ containing wheat flour at $12-13 \%$ moisture content mixed with yeast $(10: 1, w / w)$ were used for insect culture. The unsexed insect adults used in the experiments were about 7 days old.

\subsection{Extraction and Isolation}

The fresh stem bark $(1.6 \mathrm{~kg})$ of Z. armatum was roughly crushed and extracted with methanol $(20 \mathrm{~L})$ under ultrasound three times. The filtrate was concentrated into crude extract $(59.2 \mathrm{~g})$ under vacuum. The extract was crudely fractionated by silica gel column chromatography (160-200 mesh, $10.0 \times 63 \mathrm{~cm}$, $1300 \mathrm{~g}$ ), eluting with a stepwise gradient of PE (petroleum ether)/EtOAc (ethyl acetate) (60:1, 40:1, 20:1, 10:1, 5:1, 2:1 and 1:1), and then $\mathrm{CHCl}_{3} / \mathrm{CH}_{3} \mathrm{OH}(30: 1,20: 1,10: 1,5: 1,1: 1$ and $\mathrm{MeOH})$ to receive 108 fractions. In total, 11 lignans were isolated from the stem bark of $Z$. armatum. The chemical isolation scheme of $Z$. armatum methanol extract is shown in Figure 3. All purified products were analyzed by ${ }^{1} \mathrm{H}$ and ${ }^{13} \mathrm{C}-\mathrm{NMR}$ spectra and then compared with literature values. Accordingly, lignans 1-11 were characterized and identified as asarinin (1) [35], fargesin (2) [31], horsfieldin (3) [36], eudesmin (4) [37], planispine A (5) [36], planispine B (6) [38], yangambin (7) [39], acuminatolide (8) [40], salicifoliol (9) [41], magnolone (10) [42], and magnone A (11) [43].

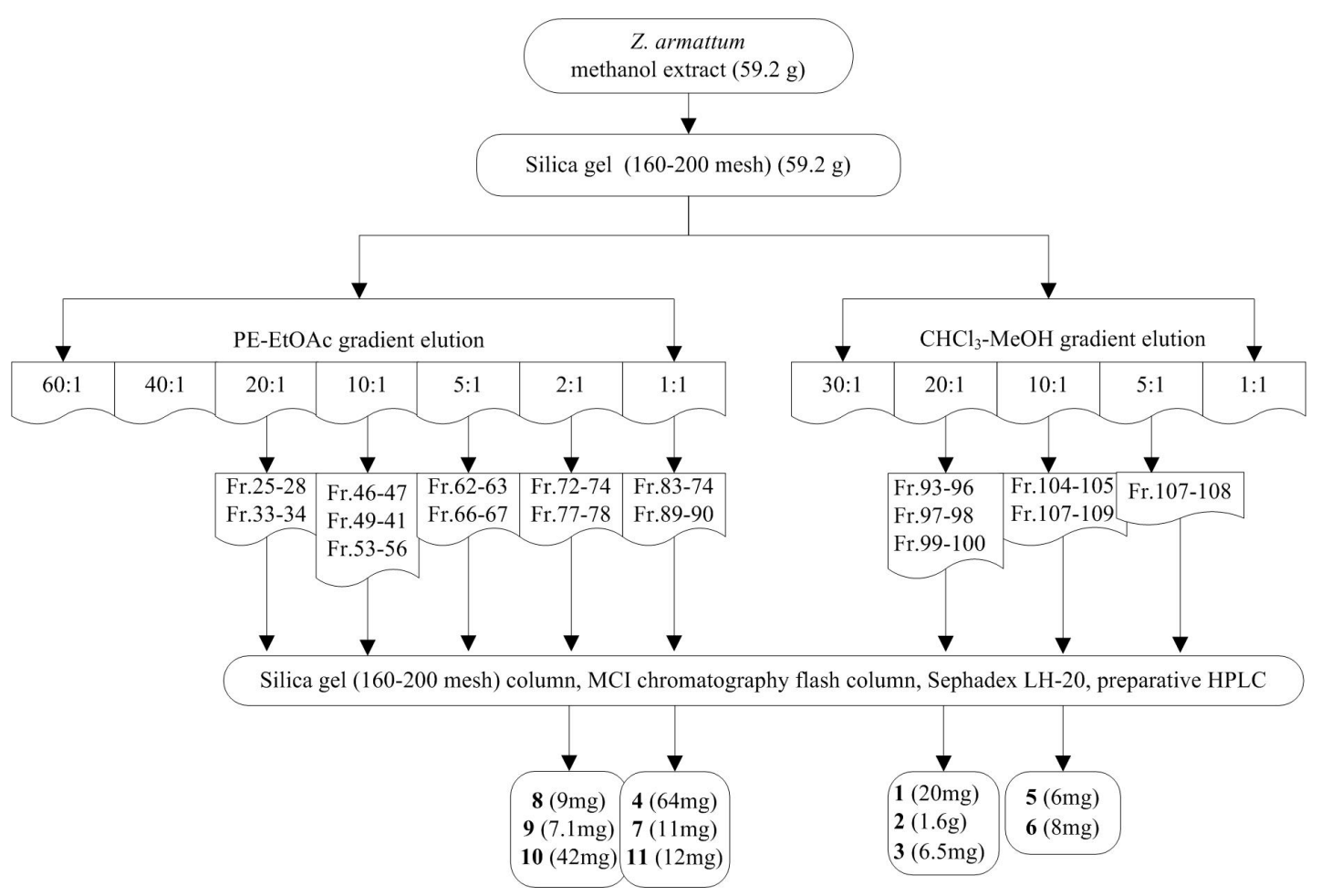

Figure 3. Chemical isolation scheme of Zanthoxylum armatum methanol extract.

Asarinin (1). White powder. ${ }^{1} \mathrm{H}-\mathrm{NMR}\left(500 \mathrm{MHz}, \mathrm{CDCl}_{3}\right) \delta$ ppm: $6.89\left(2 \mathrm{H}, \mathrm{s}, \mathrm{H}-2,2^{\prime}\right), 6.85 \sim 6.79(4 \mathrm{H}$, m, H-5, 5' ,6, 6' $), 5.98\left(2 \mathrm{H}, \mathrm{s},-\mathrm{O}-\mathrm{CH}_{2}-\mathrm{O}-\right), 5.97\left(2 \mathrm{H}, \mathrm{s},-\mathrm{O}-\mathrm{CH}_{2}-\mathrm{O}-\right), 4.85\left(1 \mathrm{H}, \mathrm{s}, \mathrm{H}-7^{\prime}\right), 4.42(1 \mathrm{H}, \mathrm{s}, 7.0 \mathrm{~Hz}$, $\mathrm{H}-7), 4.12\left(1 \mathrm{H}, \mathrm{s}, \mathrm{H}-9^{\prime} \alpha\right), 3.85\left(2 \mathrm{H}, \mathrm{m}, \mathrm{H}-9 \alpha, 9^{\prime} \beta\right), 3.32\left(2 \mathrm{H}, \mathrm{m}, \mathrm{H}-9 \beta, 8^{\prime}\right), 2.88(1 \mathrm{H}, \mathrm{m}, \mathrm{H}-8) ;{ }^{13} \mathrm{C}-\mathrm{NMR}$ 
(500 MHz, $\left.\mathrm{CDCl}_{3}\right) \delta$ ppm: $147.9\left(\mathrm{C}-4^{\prime}\right), 147.6(\mathrm{C}-4), 147.2(\mathrm{C}-3), 146.6\left(\mathrm{C}-3^{\prime}\right), 135.1(\mathrm{C}-1), 132.3\left(\mathrm{C}-1^{\prime}\right)$, 119.6 (C-6), 118.7 (C-6'), 108.2 (C-5'), 106.6 (C-2'), 106.4 (C-2), 101.1 (-O-CH$\left.{ }_{2}-\mathrm{O}-\right), 101.0$ (-O-CH 87.7 (C-7), $82.0\left(\mathrm{C}-7^{\prime}\right), 70.9\left(\mathrm{C}-9^{\prime}\right), 69.9$ (C-9), 54.7 (C-8), $50.2\left(\mathrm{C}-8^{\prime}\right)$.

Fargesin (2). Colorless cluster crystals. ${ }^{1} \mathrm{H}-\mathrm{NMR}\left(500 \mathrm{MHz}, \mathrm{CDCl}_{3}\right) \delta \mathrm{ppm}: 6.95(1 \mathrm{H}, \mathrm{s}, \mathrm{H}-2), 6.90(1 \mathrm{H}$, s, H-2' $), 6.88(2 \mathrm{H}, \mathrm{s}, \mathrm{H}-5,6), 6.85\left(1 \mathrm{H}, \mathrm{d}, J=8.0 \mathrm{~Hz}, \mathrm{H}-6^{\prime}\right), 6.80\left(1 \mathrm{H}, \mathrm{d}, J=8.0 \mathrm{~Hz}, \mathrm{H}-5^{\prime}\right), 5.97(2 \mathrm{H}, \mathrm{s}$, $\left.-\mathrm{OCH}_{2} \mathrm{O}-\right), 4.89(1 \mathrm{H}, \mathrm{d}, J=5.0 \mathrm{~Hz}, \mathrm{H}-7), 4.44\left(1 \mathrm{H}, \mathrm{d}, J=7.0 \mathrm{~Hz}, \mathrm{H}-7^{\prime}\right), 4.14\left(1 \mathrm{H}, \mathrm{d}, J=9.5 \mathrm{~Hz}, \mathrm{H}-9^{\prime} \alpha\right)$, $3.93\left(3 \mathrm{H}, \mathrm{s}, 3-\mathrm{OCH}_{3}\right), 3.90\left(3 \mathrm{H}, \mathrm{s}, 4-\mathrm{OCH}_{3}\right), 3.86\left(2 \mathrm{H}, \mathrm{dd}, J=8.0 \mathrm{~Hz}, J=7.0 \mathrm{~Hz}, \mathrm{H}-9 \alpha, 9^{\prime} \beta\right), 3.44(2 \mathrm{H}, \mathrm{m}$, $\mathrm{H}-9 \beta, 8), 2.90\left(1 \mathrm{H}, \mathrm{dd}, J=8.0 \mathrm{~Hz}, \mathrm{H}-8^{\prime}\right) ;{ }^{13} \mathrm{C}-\mathrm{NMR}\left(125 \mathrm{MHz}, \mathrm{CDCl}_{3}\right) \delta \mathrm{ppm}: 148.8(\mathrm{C}-3), 148.0(\mathrm{C}-4)$, $148.0\left(\mathrm{C}-3^{\prime}\right), 147.2\left(\mathrm{C}-4^{\prime}\right), 135.2\left(\mathrm{C}-1^{\prime}\right), 130.9$ (C-1), 119.6 (C-6'), 117.7 (C-6), 111.0 (C-5), 109.0 (C-2), 108.2 $\left(\mathrm{C}-5^{\prime}\right), 106.5\left(\mathrm{C}-2^{\prime}\right), 101.1\left(-\mathrm{OCH}_{2} \mathrm{O}-\right), 87.7\left(\mathrm{C}-7^{\prime}\right), 82.0(\mathrm{C}-7), 71.0\left(\mathrm{C}-9^{\prime}\right), 69.8(\mathrm{C}-9), 55.9\left(3-\mathrm{OCH}_{3}\right), 55.9$ $\left(4-\mathrm{OCH}_{3}\right), 54.6\left(\mathrm{C}-8^{\prime}\right), 50.2(\mathrm{C}-8)$.

Horsfieldin (3). Colorless needle crystals. ${ }^{1} \mathrm{H}-\mathrm{NMR}\left(500 \mathrm{MHz}, \mathrm{CDCl}_{3}\right) \delta \mathrm{ppm}$ : $6.97(1 \mathrm{H}, \mathrm{s}, \mathrm{H}-2), 6.91$ $\left(1 \mathrm{H}, \mathrm{d}, J=8.0 \mathrm{~Hz}, \mathrm{H}-2^{\prime}\right), 6.90(1 \mathrm{H}, \mathrm{s}, \mathrm{H}-5), 6.85\left(1 \mathrm{H}, \mathrm{d}, J=8.0 \mathrm{~Hz}, \mathrm{H}-5^{\prime}\right), 6.80\left(2 \mathrm{H}, \mathrm{d}, J=8.0 \mathrm{~Hz}, \mathrm{H}-6,6^{\prime}\right)$, $5.98\left(2 \mathrm{H}, \mathrm{s},-\mathrm{OCH}_{2} \mathrm{O}-\right), 5.60(1 \mathrm{H}, \mathrm{s}, 3-\mathrm{OH}), 4.87\left(1 \mathrm{H}, \mathrm{d}, J=5.0 \mathrm{~Hz}, \mathrm{H}-7^{\prime}\right), 4.44(1 \mathrm{H}, \mathrm{d}, J=7.0 \mathrm{~Hz}, \mathrm{H}-7)$, $4.14\left(1 \mathrm{H}, \mathrm{d}, J=9.5 \mathrm{~Hz}, \mathrm{H}-9^{\prime} \alpha\right), 3.94\left(3 \mathrm{H}, \mathrm{s}, 4-\mathrm{OCH}_{3}\right), 3.86\left(2 \mathrm{H}, \mathrm{m}, \mathrm{H}-9 \beta, 9^{\prime} \beta\right), 3.33\left(2 \mathrm{H}, \mathrm{m}, \mathrm{H}-9 \alpha, 8^{\prime}\right), 2.89$ $(1 \mathrm{H}, \mathrm{m}, \mathrm{H}-8) ;{ }^{13} \mathrm{C}-\mathrm{NMR}\left(125 \mathrm{MHz}, \mathrm{CDCl}_{3}\right) \delta \mathrm{ppm}: 148.0\left(\mathrm{C}-3^{\prime}\right), 147.2(\mathrm{C}-4), 146.4(\mathrm{C}-3), 144.6\left(\mathrm{C}-4^{\prime}\right)$, 135.2 (C-1), $130.3\left(\mathrm{C}-1^{\prime}\right), 119.6$ (C-6), $118.4\left(\mathrm{C}-6^{\prime}\right), 114.2(\mathrm{C}-2), 108.3(\mathrm{C}-5), 108.2\left(\mathrm{C}-5^{\prime}\right), 106.6\left(\mathrm{C}-2^{\prime}\right), 101.1$ $\left(-\mathrm{OCH}_{2} \mathrm{O}-\right), 87.7(\mathrm{C}-7), 82.0\left(\mathrm{C}-7^{\prime}\right), 71.0\left(\mathrm{C}-9^{\prime}\right), 69.8(\mathrm{C}-9), 56.0\left(4-\mathrm{OCH}_{3}\right), 54.6(\mathrm{C}-8), 50.2\left(\mathrm{C}-8^{\prime}\right)$.

Eudesmin (4). Colorless cluster crystals. ${ }^{1} \mathrm{H}-\mathrm{NMR}\left(500 \mathrm{MHz}, \mathrm{CDCl}_{3}\right) \delta \mathrm{ppm}: 6.93\left(2 \mathrm{H}, \mathrm{s}, \mathrm{H}-2,2^{\prime}\right), 6.90$ $\left(2 \mathrm{H}, \mathrm{d}, J=8.0 \mathrm{~Hz}, \mathrm{H}-5,5^{\prime}\right), 6.87\left(2 \mathrm{H}, \mathrm{d}, J=8.0 \mathrm{~Hz}, \mathrm{H}-6,6^{\prime}\right), 4.78\left(2 \mathrm{H}, \mathrm{t}, J=3.0 \mathrm{~Hz}, \mathrm{H}-7,7^{\prime}\right), 4.28(2 \mathrm{H}, \mathrm{t}, \mathrm{d}$, $\left.J=8.0 \mathrm{~Hz}, \mathrm{H}-9 \alpha, 9^{\prime} \alpha\right), 3.92\left(8 \mathrm{H}, \mathrm{s}, 3,3^{\prime}-\mathrm{OCH}_{3}, \mathrm{H}-9 \beta, 9^{\prime} \beta\right), 3.90\left(6 \mathrm{H}, \mathrm{s}, 4,4^{\prime}-\mathrm{OCH}_{3}\right), 3.14\left(2 \mathrm{H}, \mathrm{s}, \mathrm{H}-8,8^{\prime}\right)$, ${ }^{13} \mathrm{C}-\mathrm{NMR}\left(125 \mathrm{MHz}, \mathrm{CDCl}_{3}\right) \delta$ ppm: $149.2\left(\mathrm{C}-3,3^{\prime}\right), 148.6\left(\mathrm{C}-4,4^{\prime}\right), 133.6\left(\mathrm{C}-1,1^{\prime}\right), 118.3\left(\mathrm{C}-6,6^{\prime}\right), 111.0$ $\left(\mathrm{C}-2,2^{\prime}\right), 109.2\left(\mathrm{C}-5,5^{\prime}\right), 85.8\left(\mathrm{C}-7,7^{\prime}\right), 71.7\left(\mathrm{C}-9,9^{\prime}\right), 56.0\left(\mathrm{C}-3,3^{\prime}\right), 55.9\left(\mathrm{C}-4,4^{\prime}\right), 54.2\left(\mathrm{C}-8,8^{\prime}\right)$.

Planispine A (5). Colorless oil. ${ }^{1} \mathrm{H}-\mathrm{NMR}\left(500 \mathrm{MHz}, \mathrm{CDCl}_{3}\right) \delta \mathrm{ppm}: 6.98(1 \mathrm{H}, \mathrm{s}, \mathrm{H}-6), 6.94\left(1 \mathrm{H}, \mathrm{s}, \mathrm{H}-6^{\prime}\right)$, $6.91(1 \mathrm{H}, \mathrm{d}, J=8.5 \mathrm{~Hz}, \mathrm{H}-5), 6.88\left(2 \mathrm{H}, \mathrm{d}, \mathrm{H}-5^{\prime}, 2\right), 6.81\left(1 \mathrm{H}, \mathrm{d}, J=8.0 \mathrm{~Hz}, \mathrm{H}-2^{\prime}\right), 5.61\left(1 \mathrm{H}, \mathrm{s}, 4^{\prime}-\mathrm{OH}\right)$, $5.54\left(1 \mathrm{H}, \mathrm{t}, J=6.0 \mathrm{~Hz}, \mathrm{H}-2^{\prime \prime}\right), 4.88(1 \mathrm{H}, \mathrm{d}, J=5.0 \mathrm{~Hz}, \mathrm{H}-7), 4.60\left(2 \mathrm{H}, \mathrm{d}, J=6.5 \mathrm{~Hz}, \mathrm{H}-1^{\prime \prime}\right), 4.46(1 \mathrm{H}, \mathrm{d}$, $J=7.0 \mathrm{~Hz}, \mathrm{H}-8), 4.15(1 \mathrm{H}, \mathrm{d}, J=7.0 \mathrm{~Hz}, \mathrm{H}-9 \alpha), 3.94\left(3 \mathrm{H}, \mathrm{s}, 3^{\prime}-\mathrm{OCH}_{3}\right), 3.91\left(3 \mathrm{H}, \mathrm{s}, 3-\mathrm{OCH}_{3}\right), 3.87(2 \mathrm{H}, \mathrm{t}$, $\left.J=7.0 \mathrm{~Hz}, \mathrm{H}-9 \beta, 9^{\prime} \beta\right), 3.34\left(2 \mathrm{H}, \mathrm{d}, J=5.0 \mathrm{~Hz}, \mathrm{H}-8,9^{\prime} \alpha\right), 2.94\left(1 \mathrm{H}, \mathrm{m}, \mathrm{H}-8^{\prime}\right) ;{ }^{13} \mathrm{C}-\mathrm{NMR}\left(125 \mathrm{MHz}, \mathrm{CDCl}_{3}\right)$ $\delta$ ppm: 149.7 (C-3), 148.0 (C-4), $146.4\left(\mathrm{C}-3^{\prime}\right), 144.6$ (C-4'), 137.6 (C-1), 133.7 (C-1'), 130.4 (C-6), 120.0 (C-2"), $118.4\left(\mathrm{C}-6^{\prime}\right), 118.4(\mathrm{C}-5), 114.2\left(\mathrm{C}-5^{\prime}\right), 112.9\left(\mathrm{C}-2^{\prime}\right), 109.3\left(\mathrm{C}-3^{\prime \prime}\right), 108.4(\mathrm{C}-2), 87.7\left(\mathrm{C}-7^{\prime}\right), 82.1$ (C-7), $71.0(\mathrm{C}-9), 69.7\left(\mathrm{C}-9^{\prime}\right), 65.8\left(\mathrm{C}-1^{\prime \prime}\right), 56.0\left(3^{\prime}-\mathrm{OCH}_{3}\right), 55.9\left(3-\mathrm{OCH}_{3}\right), 54.4\left(\mathrm{C}-8^{\prime}\right), 50.2(\mathrm{C}-8), 25.8\left(\mathrm{C}-4^{\prime \prime}\right)$, $18.2\left(\mathrm{C}-5^{\prime \prime}\right)$.

Planispine B (6). Colorless oil. ${ }^{1} \mathrm{H}-\mathrm{NMR}\left(500 \mathrm{MHz}, \mathrm{CDCl}_{3}\right) \delta$ ppm: $6.92 \sim 6.86\left(6 \mathrm{H}, \mathrm{m}, \mathrm{H}-2,2^{\prime}, 5,6,6^{\prime}, 5^{\prime}\right)$, $5.53\left(1 \mathrm{H}, \mathrm{t}, J=5.5 \mathrm{~Hz}, \mathrm{H}-2^{\prime \prime}\right), 5.10\left(1 \mathrm{H}, \mathrm{t}, J=7.0 \mathrm{~Hz}, \mathrm{H}-7^{\prime \prime}\right), 4.77(2 \mathrm{H}, \mathrm{dd}, J=4.5 \mathrm{~Hz}$, $\left.J=7.5 \mathrm{~Hz}, \mathrm{H}-7,7^{\prime}\right), 4.63\left(1 \mathrm{H}, \mathrm{d}, J=4.5 \mathrm{~Hz}, \mathrm{H}-1^{\prime \prime}\right), 4.28\left(2 \mathrm{H}, \mathrm{t}, J=8.0 \mathrm{~Hz}, \mathrm{H}-9^{\prime}\right), 3.93\left(3 \mathrm{H}, \mathrm{s}, 3-\mathrm{OCH}_{3}\right), 3.91$ $\left(5 \mathrm{H}, \mathrm{s}, 3^{\prime}-\mathrm{OCH}_{3}, \mathrm{H}-9\right), 3.14\left(2 \mathrm{H}, \mathrm{m}, \mathrm{H}-8,8^{\prime}\right), 2.13\left(2 \mathrm{H}, \mathrm{t}, J=8.0 \mathrm{~Hz}, \mathrm{H}-5^{\prime \prime}\right), 2.07\left(2 \mathrm{H}, \mathrm{m}, \mathrm{H}-6^{\prime \prime}\right), 1.74(3 \mathrm{H}, \mathrm{s}$, H-4"), 1.69 (3H, s, H-9"), 1.62 (3H, s, H-10"); ${ }^{13} \mathrm{C}-\mathrm{NMR}\left(125 \mathrm{MHz}, \mathrm{CDCl}_{3}\right) \delta$ ppm: 149.7 (C-3), 147.9 (C-4), $146.7\left(\mathrm{C}-3^{\prime}\right), 145.3\left(\mathrm{C}-4^{\prime}\right), 140.6\left(\mathrm{C}-3^{\prime \prime}\right), 133.5\left(\mathrm{C}-1^{\prime}\right), 132.9(\mathrm{C}-1), 131.8\left(\mathrm{C}-8^{\prime \prime}\right), 123.9\left(\mathrm{C}-7^{\prime \prime}\right), 119.8$ (C-2") $119.0\left(\mathrm{C}-6^{\prime}\right), 118.2(\mathrm{C}-6), 114.3\left(\mathrm{C}-5^{\prime}\right), 113.0(\mathrm{C}-5), 109.5\left(\mathrm{C}-2^{\prime}\right), 108.6(\mathrm{C}-2), 85.9(\mathrm{C}-7), 85.8\left(\mathrm{C}-7^{\prime}\right)$, $71.7\left(\mathrm{C}-9,9^{\prime}\right), 66.0\left(\mathrm{C}-1^{\prime \prime}\right), 56.0\left(3,3^{\prime}-\mathrm{OCH}_{3}\right), 54.2(\mathrm{C}-8), 54.1\left(\mathrm{C}-8^{\prime}\right), 39.6\left(\mathrm{C}-5^{\prime \prime}\right), 26.3\left(\mathrm{C}-6^{\prime \prime}\right), 25.7\left(\mathrm{C}-9^{\prime \prime}\right)$, $17.7\left(\mathrm{C}-10^{\prime \prime}\right), 16.7\left(\mathrm{C}-4^{\prime \prime}\right)$.

Yangambin (7). Colorless oil. ${ }^{1} \mathrm{H}-\mathrm{NMR}\left(500 \mathrm{MHz}, \mathrm{CDCl}_{3}\right) \delta \mathrm{ppm}: 6.59\left(4 \mathrm{H}, \mathrm{s}, \mathrm{H}-2,2^{\prime}, 6,6^{\prime}\right), 4.77$ $\left(2 \mathrm{H}, \mathrm{d}, J=4.0 \mathrm{~Hz}, \mathrm{H}-1,1^{\prime}\right), 4.33\left(2 \mathrm{H}, \mathrm{dd}, J=9.0 \mathrm{~Hz}, J=7.0 \mathrm{~Hz}, \mathrm{H}-9 \alpha, 9^{\prime} \alpha\right), 3.95(2 \mathrm{H}, \mathrm{dd}, J=9.0 \mathrm{~Hz}$, $\left.J=3.5 \mathrm{~Hz}, \mathrm{H}-9 \beta, 9^{\prime} \beta\right), 3.90\left(12 \mathrm{H}, \mathrm{s}, 3,3^{\prime}, 5,5^{\prime}-\mathrm{OCH}_{3}\right), 3.86\left(6 \mathrm{H}, \mathrm{s}, 4,4^{\prime}-\mathrm{OCH}_{3}\right), 3.13\left(2 \mathrm{H}, \mathrm{m}, \mathrm{H}-8,8^{\prime}\right)$; ${ }^{13} \mathrm{C}-\mathrm{NMR}\left(125 \mathrm{MHz}, \mathrm{CDCl}_{3}\right) \delta$ ppm: $153.5\left(\mathrm{C}-3,3^{\prime}, 5,5^{\prime}\right), 137.5\left(\mathrm{C}-4,4^{\prime}\right), 136.7\left(\mathrm{C}-1,1^{\prime}\right), 102.8\left(\mathrm{C}-2,2^{\prime}, 6,6^{\prime}\right)$, $86.0\left(\mathrm{C}-7,7^{\prime}\right), 72.0\left(\mathrm{C}-9,9^{\prime}\right), 60.9\left(4,4^{\prime}-\mathrm{OCH}_{3}\right), 56.2\left(3,3^{\prime}, 5,5^{\prime}-\mathrm{OCH}_{3}\right), 54.4\left(\mathrm{C}-8,8^{\prime}\right)$. 
Acuminatolide (8). Colorless needle crystals. ${ }^{1} \mathrm{H}-\mathrm{NMR}\left(500 \mathrm{MHz}, \mathrm{CDCl}_{3}\right) \delta \mathrm{ppm}$ : $6.86(1 \mathrm{H}, \mathrm{s}, \mathrm{H}-6)$, $6.82(1 \mathrm{H}, \mathrm{s}, \mathrm{H}-2), 6.81(1 \mathrm{H}, \mathrm{s}, \mathrm{H}-3), 5.99(2 \mathrm{H}, \mathrm{s}, \mathrm{H}-10), 4.62(1 \mathrm{H}, \mathrm{d}, J=7.0 \mathrm{~Hz}, \mathrm{H}-7), 4.51(1 \mathrm{H}, \mathrm{m}, \mathrm{H}-9 \beta)$, $4.38\left(1 \mathrm{H}, \mathrm{d}, J=7.0 \mathrm{~Hz}, \mathrm{H}-9^{\prime} \beta\right), 4.35(1 \mathrm{H}, \mathrm{m}, \mathrm{H}-9 \alpha), 4.21\left(1 \mathrm{H}, \mathrm{dd}, J=3.5 \mathrm{~Hz}, J=9.5 \mathrm{~Hz}, \mathrm{H}-9^{\prime} \alpha\right), 3.45(1 \mathrm{H}$, $\mathrm{td}, J=3.5 \mathrm{~Hz}, J=9.5 \mathrm{~Hz}, \mathrm{H}-8), 3.10\left(1 \mathrm{H}, \mathrm{m}, \mathrm{H}-8^{\prime}\right) ;{ }^{13} \mathrm{C}-\mathrm{NMR}\left(125 \mathrm{MHz}, \mathrm{CDCl}_{3}\right) \delta \mathrm{ppm:} 178.1\left(\mathrm{C}-7^{\prime}\right)$, 148.3 (C-5), 147.8 (C-4), 132.8 (C-1), 119.6 (C-2), 108.4 (C-3), 106.4 (C-6), $101.3\left(-\mathrm{OCH}_{2} \mathrm{O}-\right), 86.1$ (C-7), $70.1(\mathrm{C}-9), 69.8\left(\mathrm{C}-9^{\prime}\right), 48.4(\mathrm{C}-8), 46.0\left(\mathrm{C}-8^{\prime}\right)$.

Salicifoliol (9). Colorless oil. ${ }^{1} \mathrm{H}-\mathrm{NMR}\left(500 \mathrm{MHz}, \mathrm{CDCl}_{3}\right) \delta$ ppm: $6.93(1 \mathrm{H}, \mathrm{d}, J=8.0 \mathrm{~Hz}, \mathrm{H}-3), 6.90$ $(1 \mathrm{H}, \mathrm{s}, \mathrm{H}-6), 6.83(1 \mathrm{H}, \mathrm{d}, J=8.0 \mathrm{~Hz}, \mathrm{H}-2), 5.68(1 \mathrm{H}, \mathrm{s}, 4-\mathrm{OH}), 4.64(1 \mathrm{H}, \mathrm{d}, J=7.0 \mathrm{~Hz}, \mathrm{H}-7), 4.52(1 \mathrm{H}, \mathrm{dd}$, $J=9.0 \mathrm{~Hz}, J=7.0 \mathrm{~Hz}, \mathrm{H}-9 \beta), 4.39(1 \mathrm{H}, \mathrm{t}, J=9.0 \mathrm{~Hz}, \mathrm{H}-8 \beta), 4.35(1 \mathrm{H}, \mathrm{d}, J=11.0 \mathrm{~Hz}, \mathrm{H}-9 \alpha), 4.21(1 \mathrm{H}, \mathrm{dd}$, $J=9.0 \mathrm{~Hz}, J=3.5 \mathrm{~Hz}, \mathrm{H}-8 \alpha), 3.93\left(3 \mathrm{H}, \mathrm{s}, 5-\mathrm{OCH}_{3}\right), 3.47(1 \mathrm{H}, \mathrm{td}, J=9.0 \mathrm{~Hz}, J=3.5 \mathrm{~Hz}, \mathrm{H}-12), 3.14(1 \mathrm{H}$, m, H-11); ${ }^{13} \mathrm{C}-\mathrm{NMR}\left(125 \mathrm{MHz}, \mathrm{CDCl}_{3}\right) \delta$ ppm: 178.2 (C-10), 146.9 (C-5), 145.9 (C-4), 130.6 (C-1), 119.1 (C-2), 114.4 (C-3), 108.5 (C-6), 86.1 (C-7), 70.0 (C-8), 69.9 (C-9), 56.1 (5-OCH 3 ), 48.2 (C-11), 46.0 (C-12).

Magnolone (10). White powder. ${ }^{1} \mathrm{H}-\mathrm{NMR}\left(500 \mathrm{MHz}, \mathrm{CDCl}_{3}\right) \delta \mathrm{ppm}$ : $7.62\left(1 \mathrm{H}, \mathrm{d}, J=8.5 \mathrm{~Hz}, \mathrm{H}-6^{\prime}\right), 7.59$ $\left(1 \mathrm{H}, \mathrm{s}, \mathrm{H}-2^{\prime}\right), 6.99(1 \mathrm{H}, \mathrm{s}, \mathrm{H}-2), 6.93\left(\mathrm{~d}, 2 \mathrm{H}, J=8.5 \mathrm{~Hz}, \mathrm{H}-5^{\prime}\right), 6.88(1 \mathrm{H}, \mathrm{d}, J=8.0 \mathrm{~Hz}, \mathrm{H}-6), 6.79(1 \mathrm{H}, \mathrm{d}$, $J=8.0 \mathrm{~Hz}, \mathrm{H}-5), 5.97\left(2 \mathrm{H}, \mathrm{s},-\mathrm{OCH}_{2} \mathrm{O}-\right), 4.70(2 \mathrm{H}, \mathrm{d}, J=9.0 \mathrm{~Hz}, \mathrm{H}-7), 4.31\left(1 \mathrm{H}, \mathrm{m}, \mathrm{H}-9^{\prime} \beta\right), 4.18(1 \mathrm{H}, \mathrm{m}$, H-8 $\left.{ }^{\prime}\right), 4.18\left(1 \mathrm{H}, \mathrm{m}, \mathrm{H}-9^{\prime} \alpha\right), 3.98\left(1 \mathrm{H}, \mathrm{s}, 3^{\prime}-\mathrm{OCH}_{3}\right), 3.96\left(1 \mathrm{H}, \mathrm{s}, 4^{\prime}-\mathrm{OCH}_{3}\right), 3.79(1 \mathrm{H}, \mathrm{dd}, \mathrm{H}-9 \beta), 3.68(1 \mathrm{H}$, dd, H-9 $\alpha), 2.88$ (1H, m, H-8); ${ }^{13} \mathrm{C}-\mathrm{NMR}\left(125 \mathrm{MHz}, \mathrm{CDCl}_{3}\right) \delta$ ppm: $197.8\left(\mathrm{C}-7^{\prime}\right), 153.7\left(\mathrm{C}-4^{\prime}\right), 149.3\left(\mathrm{C}-3^{\prime}\right)$, 148.0 (C-3), 147.4 (C-4), 134.5 (C-1), $129.8\left(\mathrm{C}-1^{\prime}\right), 123.2\left(\mathrm{C}-6^{\prime}\right), 120.4(\mathrm{C}-6), 110.6\left(\mathrm{C}-2^{\prime}\right), 110.1\left(\mathrm{C}-5^{\prime}\right), 108.1$ (C-5), $107.1(\mathrm{C}-2), 101.1\left(-\mathrm{OCH}_{2} \mathrm{O}-\right), 83.7(\mathrm{C}-7), 70.9\left(\mathrm{C}-9^{\prime}\right), 61.3(\mathrm{C}-9), 56.1\left(3^{\prime}-\mathrm{OCH}_{3}\right), 56.0\left(4^{\prime}-\mathrm{OCH}_{3}\right)$, $52.4(\mathrm{C}-8), 49.6\left(\mathrm{C}-8^{\prime}\right)$.

Magnone A (11). Colorless oil. ${ }^{1} \mathrm{H}-\mathrm{NMR}\left(500 \mathrm{MHz}, \mathrm{CDCl}_{3}\right) \delta \mathrm{ppm}: 7.63\left(1 \mathrm{H}, \mathrm{d}, J=9.0 \mathrm{~Hz}, \mathrm{H}-2^{\prime}\right)$, $7.60\left(1 \mathrm{H}, \mathrm{d}, J=1.5 \mathrm{~Hz}, \mathrm{H}-6^{\prime}\right), 7.05(1 \mathrm{H}, \mathrm{d}, J=1.5 \mathrm{~Hz}, \mathrm{H}-2), 6.95(1 \mathrm{H}, \mathrm{d}, J=2.0 \mathrm{~Hz}, \mathrm{H}-6), 6.93(1 \mathrm{H}, \mathrm{d}$, $\left.J=8.5 \mathrm{~Hz}, \mathrm{H}-3^{\prime}\right), 6.85(1 \mathrm{H}, \mathrm{d}, J=8.0 \mathrm{~Hz}, \mathrm{H}-5), 4.72(1 \mathrm{H}, \mathrm{d}, J=9.0 \mathrm{~Hz}, \mathrm{H}-7), 4.33\left(1 \mathrm{H}, \mathrm{m}, \mathrm{H}-9^{\prime} \beta\right), 4.21(2 \mathrm{H}$, $\left.\mathrm{m}, \mathrm{H}-8^{\prime}, 9^{\prime} \alpha\right), 3.98\left(3 \mathrm{H}, \mathrm{s}, 3-\mathrm{OCH}_{3}\right), 3.96\left(3 \mathrm{H}, \mathrm{s}, 4-\mathrm{OCH}_{3}\right), 3.94\left(3 \mathrm{H}, \mathrm{s}, 5^{\prime}-\mathrm{OCH}_{3}\right), 3.89\left(3 \mathrm{H}, \mathrm{s}, 4^{\prime}-\mathrm{OCH}_{3}\right)$, $3.80(1 \mathrm{H}, \mathrm{dd}, J=11.0 \mathrm{~Hz}, J=4.5 \mathrm{~Hz}, \mathrm{H}-9 \beta), 3.70(1 \mathrm{H}, \mathrm{dd}, J=11.0 \mathrm{~Hz}, J=4.5 \mathrm{~Hz}, \mathrm{H}-9 \alpha), 2.94(1 \mathrm{H}, \mathrm{m}$, $\mathrm{H}-8) ;{ }^{13} \mathrm{C}-\mathrm{NMR}\left(125 \mathrm{MHz}, \mathrm{CDCl}_{3}\right) \delta$ ppm: $198.0\left(\mathrm{C}-7^{\prime}\right), 153.7\left(\mathrm{C}-4^{\prime}\right), 149.3\left(\mathrm{C}-5^{\prime}\right), 149.2(\mathrm{C}-4), 148.9$ (C-3), 133.0 (C-1), $129.8\left(\mathrm{C}-1^{\prime}\right), 123.2\left(\mathrm{C}-2^{\prime}\right), 119.3$ (C-6), 110.9 (C-5), $110.6\left(\mathrm{C}-6^{\prime}\right), 110.1\left(\mathrm{C}-3^{\prime}\right), 109.6$ (C-2), 83.8 (C-7), $70.9\left(\mathrm{C}-9^{\prime}\right), 61.4(\mathrm{C}-9), 56.1\left(3-\mathrm{OCH}_{3}\right), 56.0\left(4 / 5^{\prime}-\mathrm{OCH}_{3}\right), 55.9\left(4^{\prime}-\mathrm{OCH}_{3}\right), 52.2(\mathrm{C}-8), 49.7\left(\mathrm{C}-8^{\prime}\right)$.

\subsection{Antifeedant Assay}

An antifeedant assay was performed using a modified method, as reported in References [44,45]. The compound sample was precisely weighed at $2 \mathrm{mg}$ and diluted in ethanol to fabricate the mother liquor with a concentration of $1 \mathrm{mg} / \mathrm{mL}$. Then, different volumes of the mother liquor were diluted in water to obtain different solution concentrations of $25,74,222,667$, and $2000 \mathrm{ppm}$. After that, the final solutions with different concentrations were stirred well with $0.4 \mathrm{~g}$ flour. The blank control was fabricated by the mixture of $2 \mathrm{~mL}$ water and $0.4 \mathrm{~g}$ flour. Then, $1-\mathrm{mL}$ tips were cut about $1 \mathrm{~cm}$ from the bottom to make an opening, and enlarged to about a 2-mm internal diameter. Subsequently, $200 \mu \mathrm{L}$ mixture was pipetted into a clean dish to make about 6-8 small cookies drop by drop. After being air-dried overnight, the small cookies with different concentrations were transferred into the incubator for $48 \mathrm{~h}$ to achieve equilibration at $29-30{ }^{\circ} \mathrm{C}$ and $70-80 \%$ relative humidity $(\mathrm{RH})$. Twenty unsexed adult insects were selected and placed into glass vials (diameter: $2.5 \mathrm{~cm}$, height: $5.5 \mathrm{~cm}$ ) and starved for $24 \mathrm{~h}$ for further weighing. After that, small cookies were added into each vial and weighed later. For each concentration, the test was conducted five times. Seventy-two hours later, the total weight was determined. Then, all the insects were singled out immediately, and each vial with remaining cookies were weighed again. The percent antifeedant index was calculated as follows: Antifeedant index (\%) $=[(C-T) / C] \times 100$, where $C$ is the weight of the diet consumed in the blank control and $T$ is the weight of the diet consumed in the treated groups. The antifeedant index and $\mathrm{EC}_{50}$ were calculated by SPSS (IBM) and Origin software. Statistical analysis was carried out using Student's $t$-test to compare 
the two groups, with $p<0.05$ being indicative of significance. A commercial antifeedant reagent, toosendanin, used as a positive control, was purchased from China National Standards Network.

\section{Conclusions}

Eleven lignans were isolated from the stem bark of Zanthoxylum armatum, and five of them were obtained for the first time. The antifeedant activities of these compounds were measured, and one of them, asarinin (1), showed stronger activity than the positive control. Three other compounds, fargesin (2), horsfieldin (3), and magnolone (10), exhibited the same antifeedant activities as the positive control. Furthermore, compounds that displayed high antifeedant activities have a common chemical group, namely methylenedioxy. These results indicated that compounds with one or more methylenedioxy groups have potential for development into novel antifeedants or lead compounds to protect food crops in storage.

Acknowledgments: This project was supported by the National Key Research and Development Program (2016YFC0500805), Beijing Municipal Natural Science Foundation (No. 7142093), and Fundamental Research Funds for the Central Universities.

Author Contributions: W.Z., Y.W., and S.D. conceived and designed the experiments; W.Z., Y.W., and Z.Z. performed the experiments; W.Z. and Z.G. analyzed the data; J.C., X.P., and Z.C. contributed materials and analysis tools; W.Z. wrote the paper; Z.D. revised the paper.

Conflicts of Interest: The authors declare no conflict of interest.

\section{References}

1. Hematpoor, A.; Liew, S.Y.; Azirun, M.S.; Awang, K. Insecticidal activity and the mechanism of action of three phenylpropanoids isolated from the roots of Piper sarmentosum Roxb. Sci. Rep. 2017, 7, 1-13. [CrossRef] [PubMed]

2. Rajashekar, Y.; Shivanandappa, T. A novel natural insecticide molecule for grain protection. Julius-Kühn-Archiv 2010, 910-915. [CrossRef]

3. Denell, R.; Gibbs, R.; Muzny, D.; Weinstock, G.M.; Attaway, T.; Bell, S.; Buhay, C.J.; Chandrabose, M.N.; Chavez, D.; Clerk-Blankenburg, K.P.; et al. The genome of the model beetle and pest Tribolium castaneum. Nature 2008, 452, 949-955.

4. Gokhale, C.S.; Traulsen, A.; Joop, G. Social dilemma in the external immune system of the red flour beetle? It is a matter of time. Ecol. Evol. 2017, 7, 6758-6765. [CrossRef] [PubMed]

5. Kittelmann, S.; Ulrich, J.; Posnien, N.; Bucher, G. Changes in anterior head patterning underlie the evolution of long germ embryogenesis. Dev. Biol. 2013, 374, 174-184. [CrossRef] [PubMed]

6. Cheng, S.S.; Chang, H.T.; Chang, S.T.; Tsai, K.H.; Chen, W.J. Bioactivity of selected plant essential oils against the yellow fever mosquito Aedes aegypti larvae. Bioresour. Technol. 2003, 89, 99-102. [CrossRef]

7. Zettler, J.L.; Arthur, F.H. Chemical control of stored product insects with fumigants and residual treatments. Crop Prot. 2000, 19, 577-582. [CrossRef]

8. Arthur, F.H. Grain protectants: Current status and prospects for the future. J. Stored Prod. Res. 1996, 32, 293-302. [CrossRef]

9. Adakole, J.A.; Adeyemi, A.F.F. Larvicidal effects of Cymbopogon citratus (lemon grass) extract against Culex quinquefasciatus qularvae (Diptera, culicidae). Int. J. Appl. Environ. Sci. 2012, 7, 187-192.

10. Tavares, W.S.; Costa, M.A.; Cruz, I.; Silveira, R.D.; Serrao, J.E.; Zanuncio, J.C. Selective effects of natural and synthetic insecticides on mortality of Spodoptera frugiperda (Lepidoptera: Noctuidae) and its predator Eriopis connexa (Coleoptera: Coccinellidae). J. Environ. Sci. Heal 2010, 45, 557-561. [CrossRef] [PubMed]

11. Alam, F.; Us Saqib, Q.N. Evaluation of Zanthoxylum armatum Roxb for in vitro biological activities. J. Tradit. Complement. Med. 2017, 7, 515-518. [CrossRef] [PubMed]

12. Li, H.; Li, P.; Zhu, L.; Xie, M. Studies on the chemical constituents of Zanthoxylum armatum DC. Chin. Pharm. 2006, 13, 1035-1036.

13. Chen, Y.; Hu, Y.; He, H.; Yang, G. Chemical constituents from barks of Zanthoxylum planispinum. Chin. Tradit. Herb. Drugs 2013, 44, 3429-3434. 
14. Agnihotri, S.; Wakode, S.; Ali, M. Chemical constituents isolated from Zanthoxylum armatum stem bark. Chem. Nat. Compd. 2017, 53, 880-882. [CrossRef]

15. Bhatt, V.; Sharma, S.; Kumar, N.; Singh, B. A new lignan from the Leaves of Zanthoxylum armatum. Nat. Prod. Commun. 2017, 12, 99-100.

16. Guo, T.; Dai, L.; Tang, X.; Song, T.; Wang, Y.; Zhao, A.; Cao, Y.; Chang, J. Two new phenolic glycosides from the stem of Zanthoxylum armatum DC. Nat. Prod. Res. 2017, 31, 2335-2340. [CrossRef] [PubMed]

17. Guo, T.; Li, Y. Determination of four lignans in roots, stems and leaves of Zanthoxylum armatum DC by HPLC-DAD with HPLC-ESI-QTOF-MS confirmation. J. Anal. Chem. 2016, 71, 527-533. [CrossRef]

18. Kumar, V.; Reddy, S.G.E.; Chauhan, U.; Kumar, N.; Singh, B. Chemical composition and larvicidal activity of Zanthoxylum armatum against diamondback moth, Plutella xylostella. Nat. Prod. Res. 2016, 30, 689-692. [CrossRef] [PubMed]

19. Zhou, T.; Pu, B.; Jiang, H.; Liu, C. Analysis of fatty acid compositions in Zanthoxylum armatum DC. oil with different extraction methods. J. Nucl. Agric. Sci. 2015, 29, 1323-1328.

20. Wang, C.; Zhang, W.; You, C.; Guo, S.; Geng, Z.; Fan, L.; Du, S.; Deng, Z.; Wang, Y. Insecticidal Constituents of Essential Oil Derived from Zanthoxylum armatum against Two Stored-Product Insects. J. Oleo Sci. 2015, 64, 861-868. [CrossRef] [PubMed]

21. Wang, C.; Pu, B.; Jiang, Y.; Fu, B. Extraction and antioxidant activity in vitro of flavonoids in cold pressed cake of Zanthoxylum armatum DC. Prod. Food Mach. 2017, 33, 137-142.

22. Xu, L.; Pu, B.; Jiang, Y.; Tan, X.; Wang, S.; Wang, C.; Zhang, L.; Sun, P. Studies on the antioxidatant activities of water extract from Zanthoxylum armatum DC. Prodr. in vitro. Food Ferment. Ind. 2016, 42, 140-143.

23. Zhou, T.; Pu, B.; Jiang, H.; Liu, C. Study on antibacterial activity and stability of Zanthoxylum armatum DC. Prodr. oil. Sci. Technol. Food Ind. 2015, 36, 106-109.

24. Alam, F.; Saqib, Q.N.U.; Waheed, A. Cytotoxic activity of extracts and crude saponins from Zanthoxylum armatum DC. against human breast (MCF-7, MDA-MB-468) and colorectal (Caco-2) cancer cell lines. BMC Complement. Altern. Med. 2017, 17, 1-9. [CrossRef] [PubMed]

25. Singh, T.D.; Meitei, H.T.; Sharma, A.L.; Robinson, A.; Singh, L.S.; Singh, T.R. Anticancer properties and enhancement of therapeutic potential of cisplatin by leaf extract of Zanthoxylum armatum DC. Biol. Res. 2015, 48, 1-9. [CrossRef] [PubMed]

26. Singh, G.; Singh, R.; Verma, P.K.; Singh, R.; Anand, A. Anthelmintic efficacy of aqueous extract of Zanthoxylum armatum DC. seeds against Haemonchus contortus of small ruminants. J. Parasit. Dis. Off. Organ Indian Soc. Parasitol. 2016, 40, 528-532. [CrossRef] [PubMed]

27. Teponno, R.B.; Kusari, S.; Spiteller, M. Recent advances in research on lignans and neolignans. Nat. Prod. Rep. 2016, 33, 1044-1092. [CrossRef] [PubMed]

28. Rezanka, T.; Vitova, M.; Kolouchova, I.; Nedbalova, L.; Dolezalova, J.; Palyzova, A.; Sigler, K. Polydatin and its derivatives inhibit fatty acid desaturases in microorganisms. Eur. J. Lipid Sci. Technol. 2017, 119, 1-11. [CrossRef]

29. Su, G.; Cheng, Y.; Wang, K.; Wang, X.; Wu, B. An unusual tetrahydrofuran lignan from the roots of Zanthoxylum planispinum and the potential anti-inflammatory effects. Chem. Biodivers. 2017, 14, 1-6. [CrossRef] [PubMed]

30. Papadopoulos, A.G.; Nenadis, N.; Sigalas, M.P. DFT study of radical scavenging activity of sesame oil lignans and selected in vivo metabolites of sesamin. Comput. Theor. Chem. 2016, 1077, 125-132. [CrossRef]

31. Messiano, G.B.; Vieira, L.; Machado, M.B.; Lopes, L.M.X.; De Bortoli, S.A.; Zukerman-Schpector, J. Evaluation of insecticidal activity of diterpenes and lignans from Aristolochia malmeana against Anticarsia gemmatalis. J. Agric. Food Chem. 2008, 56, 2655-2659. [CrossRef] [PubMed]

32. Woo, K.W.; Suh, W.S.; Subedi, L.; Kim, S.Y.; Kim, A.; Lee, K.R. Bioactive lignan derivatives from the stems of Firmiana simplex. Bioorg. Med. Chem. Lett. 2016, 26, 730-733. [CrossRef] [PubMed]

33. Yin, J.; Han, N.; Liu, Z.; Xu, X.; Zhang, B.; Kadota, S. The in vitro anti-osteoporotic activity of some diarylheptanoids and lignans from the rhizomes of Dioscorea spongiosa. Planta Med. 2008, 74, 1451-1453. [CrossRef] [PubMed]

34. Ma, Z.; Zhang, J.; Yang, L.; Ma, J. Analysis and comparison of the volatile oil from different parts of Zanthoxylum ovalifolium var. spinifolium by GC-MS. Chin. Pharm. J. 2004, 39, 502-503. 
35. Jing, Y.; Zhang, Y.; Shang, M.; Yu, J.; Tang, J.; Liu, G.; Li, Y.; Li, X.; Wang, X.; Cai, S. Phenanthrene derivatives from roots and rhizomes of Asarum heterotropoides var. mandshuricum. Fitoterapia 2017, 117, 101-108. [CrossRef] [PubMed]

36. Guo, T. Studies on the Chemical Constituents of Zanthoxylum armatum DC. and Z. avicenna (Lam.) DC. and Analgesic, Anti-Inflammatory Activites of Z. armatum; Fudan University: Fudan, China, 2011.

37. Latip, J.; Hartley, T.G.; Waterman, P.G. Lignans and coumarins metabolites from Melicope hayesii. Phytochemistry 1999, 51, 107-110. [CrossRef]

38. Hu, Y.; Chen, Y.; Shang, Y.; Yang, G. Two New lignans from the bark of Zanthoxylum planispinum. B Korean Chem. Soc. 2009, 30, 1884-1886.

39. Ahmed, A.A.; Mahmoud, A.A.; Ali, E.T.; Tzakou, O.; Couladis, M.; Mabry, T.J.; Gati, T.; Toth, G. Two highly oxygenated eudesmanes and 10 lignans from Achillea holosericea. Phytochemistry 2002, 59, 851-856. [CrossRef]

40. Saladino, R.; Fiani, C.; Crestini, C.; Argyropoulos, D.S.; Marini, S.; Coletta, M. An efficient and stereoselective dearylation of asarinin and sesamin tetrahydrofurofuran lignans to acuminatolide by methyltrioxorhenium $/ \mathrm{H}_{2} \mathrm{O}_{2}$ and UHP Systems. J. Nat. Prod. 2007, 70, 39-42. [CrossRef] [PubMed]

41. Aguiar, R.M.; Alves, C.Q.; David, J.M.; de Rezende, L.C.; Lima, L.S.; David, J.P.; de Queiroz, L.P. Antioxidant activities of isolated compounds from stems of Mimosa invisa mart. ex colla. Quim. Nova 2012, 35, 567-570. [CrossRef]

42. Yu, H.J.; Chen, C.C.; Shieh, B.J. Two new constituents from the leaves of Magnolia coco. J. Nat. Prod. 1998, 61, 1017-1019. [CrossRef] [PubMed]

43. Jung, K.Y.; Kim, D.S.; Oh, S.R.; Park, S.H.; Lee, I.S.; Lee, J.J.; Shin, D.H.; Lee, H.K. Magnone A and B, novel anti-PAF tetrahydrofuran lignans from the flower buds of Magnolia fargesii. J. Nat. Prod. 1998, 61, 808-811. [CrossRef] [PubMed]

44. Du, S.S.; Wang, C.F.; Li, J.; Zhang, H.M.; Liu, Q.Z.; Liu, Z.L.; Deng, Z.W. Antifeedant diterpenoids against Tribolium castaneum from the stems and twigs of Ceriops tagal (Rhizophoraceae). Molecules 2011, 16, 6060-6067. [CrossRef] [PubMed]

45. Wang, C.; You, C.; Yang, K.; Guo, S.; Geng, Z.; Fan, L.; Du, S.; Deng, Z.; Wang, Y. Antifeedant activities of methanol extracts of four Zanthoxylum species and benzophenanthridines from stem bark of Zanthoxylum schinifolium against Tribolium castaneum. Ind. Crop Prod. 2015, 74, 407-411. [CrossRef]

Sample Availability: Samples of the compounds are available from the authors.

(C) 2018 by the authors. Licensee MDPI, Basel, Switzerland. This article is an open access article distributed under the terms and conditions of the Creative Commons Attribution (CC BY) license (http://creativecommons.org/licenses/by/4.0/). 